\title{
On conservation and dual consistency for summation-by-parts based approximations of parabolic problems
}

Fatemeh Ghasemi Zinatabadi and J an Nordström

The self-archived postprint version of this journal article is available at Linköping University Institutional Repository (DiVA):

http:/ / urn.kb.se/ resolve?urn=urn:nbn:se:liu:diva-164558

N.B.: When citing this work, cite the original publication.

Ghasemi Zinatabadi, F., Nordström, J ., (2020), On conservation and dual consistency for summationby-parts based approximations of parabolic problems, J ournal of Computational Physics, 410,

109282. https://doi.org/ 10.1016/j.jcp.2020.109282

Original publication available at:

https:// doi.org/ 10.1016/j.jcp.2020.109282

Copyright: Elsevier

http:// www.elsevier.com/

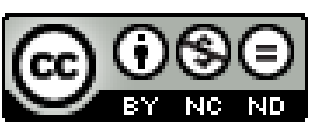




\title{
On conservation and dual consistency for summation-by-parts based approximations of parabolic problems
}

\author{
Fatemeh Ghasemi \& Jan Nordström \\ Computational Mathematics, Department of Mathematics, Linköping University, \\ SE-581 83 Linköping, Sweden \\ fatemeh.ghasemi@liu.se, jan.nordstrom@liu.se
}

\section{Introduction}

We consider the coupling of parabolic problems discretized using difference operators on summation-by-parts (SBP) form with interface conditions imposed weakly. In $[1,2]$, it was shown that conservation and dual consistency are equivalent concepts for linear conservation laws. Here, we show that these concepts are equivalent also for symmetric or symmetrizable parabolic problems, exemplified by the heat equation. We rewrite the heat equation as a first order system as in the local discontinuous Galerkin method and show the equivalence of dual consistency and conservation for both the first and second order forms $[3,4,5]$.

\section{The continuous problem}

Consider the coupling of two heat equations

$$
\begin{array}{rlrl}
u_{t} & =\left(\epsilon u_{x}\right)_{x}, & -1 \leq x \leq 0, \quad t>0, \\
v_{t} & =\left(\epsilon v_{x}\right)_{x}, & 0 \leq x \leq 1, \quad t>0, \\
u(0, t) & =v(0, t), & x & =0, \quad t>0, \\
u_{x}(0, t) & =v_{x}(0, t), & x & =0, \quad t>0,
\end{array}
$$

augmented with initial conditions. In $(1), u(x, t)$ and $v(x, t)$ are the solutions in the left and right domain, respectively. For simplicity, we assume that the thermal diffusivity $\epsilon(x, t)>0$ is continuous across the interface $x=0$. For clarity and ease of presentation, the boundary conditions at $x= \pm 1$ are ignored. 
The problem (1) is reduced to first order form by introducing the new variables $\tilde{u}=\epsilon u_{x}, \tilde{v}=\epsilon v_{x}$

$$
\begin{array}{rlrl}
\tilde{I} U_{t}+A U+B U_{x} & =0, & -1 \leq x \leq 0, & t>0, \\
\tilde{I} V_{t}+A V+B V_{x} & =0, & 0 \leq x \leq 1, \quad t>0, \\
U(0, t) & =V(0, t), & x=0, & t>0,
\end{array}
$$

augmented with homogeneous initial conditions. In (2), $U=[u, \tilde{u}]^{T}, V=$ $[v, \tilde{v}]^{T}, \tilde{I}=\operatorname{diag}(1,0), A=\operatorname{diag}(0,1 / \epsilon(x, t))$ and $B=-\left[\begin{array}{ll}0 & 1 \\ 1 & 0\end{array}\right]$.

\subsection{Conservation}

Let $\varphi(x, t) \in H^{2}((-1,1))$ be a function with compact support in $-1 \leq$ $x \leq 1$. Multiplying $(1)$ by $\varphi(x, t)$ and performing integration by parts in space twice, leads to

$$
\begin{aligned}
& \int_{-1}^{0} \varphi u_{t} d x+\int_{0}^{1} \varphi v_{t} d x=\int_{-1}^{0}\left(\epsilon \varphi_{x}\right)_{x} u d x+\int_{0}^{1}\left(\epsilon \varphi_{x}\right)_{x} v d x \\
& +\epsilon(0, t)\left(\varphi(0, t)\left(u_{x}(0, t)-v_{x}(0, t)\right)-\varphi_{x}(0, t)(u(0, t)-v(0, t))\right) .
\end{aligned}
$$

The interface conditions in (1) remove the interface terms in (3) and conservation follows.

Multiplying (2) with the transpose of the compactly supported vector function $\Phi(x, t) \in\left[H^{1}((-1,1))\right]^{2}$, and integrating in space leads to

$$
\begin{aligned}
& \int_{-1}^{0} \Phi^{T} \tilde{I} U_{t} d x+\int_{0}^{1} \Phi^{T} \tilde{I} V_{t} d x+\int_{-1}^{0} \Phi^{T} A U d x+\int_{0}^{1} \Phi^{T} A V d x \\
& =-\Phi^{T}(0, t) B(U(0, t)-V(0, t))+\int_{-1}^{0} \Phi_{x}^{T} B U d x+\int_{0}^{1} \Phi_{x}^{T} B V d x .
\end{aligned}
$$

The interface conditions in (2) remove the interface terms in (4), which (disregarding the zero order terms) implies that (2) is on conservative form.

Remark 1. In this paper, we use a broad definition of conservation where the equations are multiplied by a smooth test function. By performing integrationby-parts we transfer all derivatives to the test function. We say that the problem is conservative if all interface terms vanish. This definition is motivated by the comparison with the problem on first order form. By choosing the test functions to be unity, the usual definition of conservation is obtained. 


\subsection{The dual problem}

The dual problem [6] associated to (1) is

$$
\begin{array}{rlrl}
\phi_{\tau}=\left(\epsilon \phi_{x}\right)_{x}, & -1 \leq x \leq 0, & \tau>0, \\
\psi_{\tau}=\left(\epsilon \psi_{x}\right)_{x}, & 0 \leq x \leq 1, & \tau>0, \\
\phi(0, \tau) & =\psi(0, \tau), & x=0, & \tau>0, \\
\phi_{x}(0, \tau) & =\psi_{x}(0, \tau), & x=0, & \tau>0,
\end{array}
$$

augmented with homogeneous initial conditions at $\tau=0$. In (5), the dual time variable is $\tau=T-t$, where $T$ is the final time for the primal problem. We ignore the boundary conditions at $x= \pm 1$ as in the primal problem. In the same way as for the primal problem, (5) can be shown to be conservative. The dual problem associated to (2) is

$$
\begin{array}{rlrl}
\tilde{I} \Phi_{\tau}+A \Phi-B \Phi_{x} & =0, & -1 \leq x \leq 0, & \tau>0 \\
\tilde{I} \Psi_{\tau}+A \Psi-B \Psi_{x} & =0, & 0 \leq x \leq 1, \quad \tau>0 \\
\Phi(0, t) & =\Psi(0, t), & x=0, & \tau>0
\end{array}
$$

augmented with homogeneous initial conditions at $\tau=0$. In $(6), \Phi=[\phi, \tilde{\phi}]^{T}$ and $\Psi=[\psi, \tilde{\psi}]^{T}$. Conservation of (6) is shown in the same way as for (2).

\section{The semi-discrete approximation}

To define a semi-discretization of (1), we discretize the left and right domains by $N+1$ and $M+1$ grid points, respectively. Let the vectors $\mathbf{u}, \mathbf{v}, \mathbf{U}$ and $\mathbf{V}$ contain approximations of $u, v, U$ and $V$, respectively. Then, the first derivative is approximated using SBP operators

$$
\begin{array}{ll}
u_{x} \approx D_{L} \mathbf{u}=P_{L}^{-1} Q_{L} \mathbf{u}, & v_{x} \approx D_{R} \mathbf{v}=P_{R}^{-1} Q_{R} \mathbf{v}, \\
U_{x} \approx\left(D_{L} \otimes I_{2}\right) \mathbf{U}, & V_{x} \approx\left(D_{R} \otimes I_{2}\right) \mathbf{V}
\end{array}
$$

where $\otimes$ denotes the Kronecker product and the subscripts $L, R$ refer to the left and right spatial intervals, respectively. For simplicity, we use the first derivative twice as our second derivative operator. In (7), $P_{L, R}$ are symmetric positive definite matrices and $Q_{L, R}$ are almost skew-symmetric matrices satisfying $Q_{L, R}+Q_{L, R}^{T}=\operatorname{diag}(-1,0, \ldots, 0,1) . I_{2}$ is the $2 \times 2$ identity matrix. 
Now, we discretize (1) in space as in [5] and obtain

$$
\begin{aligned}
\mathbf{u}_{t}-D_{L}\left(\boldsymbol{\epsilon}_{L} D_{L} \mathbf{u}\right) & =P_{L}^{-1}\left[\sigma_{L}^{1}\left(u_{N}-v_{0}\right)+\sigma_{L}^{2}\left(\left(D_{L} \mathbf{u}\right)_{N}-\left(D_{R} \mathbf{v}\right)_{0}\right)\right] e_{N} \\
& +P_{L}^{-1} D_{L}^{T}\left[\sigma_{L}^{3}\left(u_{N}-v_{0}\right)+\sigma_{L}^{4}\left(\left(D_{L} \mathbf{u}\right)_{N}-\left(D_{R} \mathbf{v}\right)_{0}\right)\right] e_{N}, \\
\mathbf{v}_{t}-D_{R}\left(\boldsymbol{\epsilon}_{R} D_{R} \mathbf{v}\right) & =P_{R}^{-1}\left[\sigma_{R}^{1}\left(v_{0}-u_{N}\right)+\sigma_{R}^{2}\left(\left(D_{R} \mathbf{v}\right)_{0}-\left(D_{L} \mathbf{u}\right)_{N}\right)\right] e_{0} \\
& +P_{R}^{-1} D_{R}^{T}\left[\sigma_{R}^{3}\left(v_{0}-u_{N}\right)+\sigma_{R}^{4}\left(\left(D_{R} \mathbf{v}\right)_{0}-\left(D_{L} \mathbf{u}\right)_{N}\right)\right] e_{0},
\end{aligned}
$$

where the diagonal matrices $\boldsymbol{\epsilon}_{L}$ and $\boldsymbol{\epsilon}_{R}$ approximate $\epsilon(x, t)$ point wise on the left and right domains, respectively. In (8), the vectors $e_{N}=[0, \cdots, 0,1]^{T}$ and $e_{0}=[1,0, \cdots, 0]^{T}$ have the length of the left and right mesh, respectively. The scalars $u_{N},\left(D_{L} \mathbf{u}\right)_{N}, v_{0}$ and $\left(D_{R} \mathbf{v}\right)_{0}$ are approximations of $u, u_{x}, v$ and $v_{x}$ at $x=0$. Furthermore, the penalty terms on the right-hand side (RHS) impose the interface conditions weakly.

Next, we discretize (2) as

$$
\begin{aligned}
& \left(I_{N} \otimes \tilde{I}\right) \mathbf{U}_{t}+\left[\mathbf{A}_{L}+D_{L} \otimes B\right] \mathbf{U}=\left(P_{L}^{-1} e_{N} \otimes \Sigma_{l}\right)\left(U_{N}-V_{0}\right), \\
& \left(I_{M} \otimes \tilde{I}\right) \mathbf{V}_{t}+\left[\mathbf{A}_{R}+D_{R} \otimes B\right] \mathbf{V}=\left(P_{R}^{-1} e_{0} \otimes \Sigma_{r}\right)\left(V_{0}-U_{N}\right),
\end{aligned}
$$

where $I_{N}$ and $I_{M}$ are the identity matrices of size $N+1$ and $M+1$ and $\mathbf{A}_{L}$ and $\mathbf{A}_{R}$ approximate $A=A(x, t)$ point wise on the left and right domains, respectively.

We can rearrange the terms in (8) such that we get a first order scheme, which is equivalent to (9) if

$$
\begin{aligned}
& \Sigma_{l}=\left[\begin{array}{cc}
\sigma_{L}^{1}+\alpha_{L} \sigma_{L}^{3}-\frac{\alpha_{L}}{\beta}\left(\sigma_{L}^{2}+\alpha_{L} \sigma_{L}^{4}\right)\left(\sigma_{L}^{3}+\sigma_{R}^{3}\right) & \frac{-1}{\beta}\left(\sigma_{L}^{2}+\alpha_{L} \sigma_{L}^{4}\right) \\
\frac{-\sigma_{L}^{3}}{\epsilon_{I}}+\frac{\alpha_{L}}{\beta \epsilon_{I}} \sigma_{L}^{4}\left(\sigma_{L}^{3}+\sigma_{R}^{3}\right) & \frac{1}{\beta \epsilon_{I}} \sigma_{L}^{4}
\end{array}\right], \\
& \Sigma_{r}=\left[\begin{array}{cc}
\sigma_{R}^{1}-\alpha_{R} \sigma_{R}^{3}-\frac{\alpha_{R}}{\beta}\left(\sigma_{R}^{2}-\alpha_{R} \sigma_{R}^{4}\right)\left(\sigma_{L}^{3}+\sigma_{R}^{3}\right) & \frac{-1}{\beta}\left(\sigma_{R}^{2}-\alpha_{R} \sigma_{R}^{4}\right) \\
\frac{-\sigma_{R}^{3}}{\epsilon_{I}}+\frac{\alpha_{R}}{\beta \epsilon_{I}} \sigma_{R}^{4}\left(\sigma_{L}^{3}+\sigma_{R}^{3}\right) & \frac{1}{\beta \epsilon_{I}} \sigma_{R}^{4}
\end{array}\right],
\end{aligned}
$$

where $\epsilon_{I}=\epsilon(0, t), \alpha_{L}=e_{N}^{T} P_{L}^{-1} e_{N}, \alpha_{R}=e_{0}^{T} P_{R}^{-1} e_{0}$ and $\beta=-\epsilon_{I}+\alpha_{L} \sigma_{L}^{4}+$ $\alpha_{R} \sigma_{R}^{4}$.

Similarly, we can rewrite the scheme (9) as a second order scheme, which 
is equivalent to (8) with $\Sigma_{l}=\left[\begin{array}{cc}\sigma_{l}^{1} & \sigma_{l}^{2} \\ \sigma_{l}^{3} & \sigma_{l}^{4}\end{array}\right]$ and $\Sigma_{r}=\left[\begin{array}{cc}\sigma_{r}^{1} & \sigma_{r}^{2} \\ \sigma_{r}^{3} & \sigma_{r}^{4}\end{array}\right]$ if

$$
\begin{array}{ll}
\sigma_{L}^{1}=\sigma_{l}^{1}+\alpha_{L} \epsilon_{I} \sigma_{l}^{3}-\frac{\alpha_{L} \epsilon_{I}}{\tilde{\beta}}\left(\sigma_{l}^{2}+\alpha_{L} \epsilon_{I} \sigma_{l}^{4}\right)\left(\sigma_{l}^{3}+\sigma_{r}^{3}\right), & \sigma_{L}^{2}=-\frac{\epsilon_{I}}{\tilde{\beta}}\left(\sigma_{l}^{2}+\alpha_{L} \epsilon_{I} \sigma_{l}^{4}\right) \\
\sigma_{L}^{3}=-\epsilon_{I} \sigma_{l}^{3}+\frac{\alpha_{L} \epsilon_{I}^{2}}{\tilde{\beta}} \sigma_{l}^{4}\left(\sigma_{l}^{3}+\sigma_{r}^{3}\right), & \sigma_{L}^{4}=\frac{\epsilon_{I}^{2}}{\tilde{\beta}} \sigma_{l}^{4}, \\
\sigma_{R}^{1}=\sigma_{r}^{1}-\alpha_{R} \epsilon_{I} \sigma_{r}^{3}-\frac{\alpha_{R} \epsilon_{I}}{\tilde{\beta}}\left(\sigma_{r}^{2}-\alpha_{R} \epsilon_{I} \sigma_{r}^{4}\right)\left(\sigma_{l}^{3}+\sigma_{r}^{3}\right), & \sigma_{R}^{2}=-\frac{\epsilon_{I}}{\tilde{\beta}}\left(\sigma_{r}^{2}-\alpha_{R} \epsilon_{I} \sigma_{r}^{4}\right) \\
\sigma_{R}^{3}=-\epsilon_{I} \sigma_{r}^{3}+\frac{\alpha_{R} \epsilon_{I}^{2}}{\tilde{\beta}} \sigma_{r}^{4}\left(\sigma_{l}^{3}+\sigma_{r}^{3}\right), & \sigma_{R}^{4}=\frac{\epsilon_{I}^{2}}{\tilde{\beta}} \sigma_{r}^{4},
\end{array}
$$

where $\tilde{\beta}=-1+\alpha_{L} \epsilon_{I} \sigma_{l}^{4}+\alpha_{R} \epsilon_{I} \sigma_{l}^{4}$.

\subsection{Conservation}

Let $\varphi_{L} \in V_{L}$ and $\varphi_{R} \in V_{R}$ denote grid functions at the left and right domains where $V_{L}$ and $V_{R}$ are discrete subspaces of $H^{2}((-1,0))$ and $H^{2}((0,1))$, respectively. Multiplying (8) by $\varphi_{L}^{T} P_{L}$ and $\varphi_{R}^{T} P_{R}$ and using $\varphi_{L}^{N}=\varphi_{R}^{0}:=\varphi_{I}$ leads to

$$
\boldsymbol{\varphi}_{L}^{T} P_{L} \mathbf{u}_{t}+\boldsymbol{\varphi}_{R}^{T} P_{R} \mathbf{v}_{t}=\left[D_{L} \boldsymbol{\epsilon}_{L}\left(D_{L} \boldsymbol{\varphi}_{L}\right)\right]^{T} P_{L} \mathbf{u}+\left[D_{R} \boldsymbol{\epsilon}_{R}\left(D_{R} \boldsymbol{\varphi}_{R}\right)\right]^{T} P_{R} \mathbf{v}+I T,
$$

where

$$
\begin{aligned}
& I T=\boldsymbol{\varphi}_{I}\left(\sigma_{L}^{1}-\sigma_{R}^{1}\right)\left[u_{N}-v_{0}\right]+\boldsymbol{\varphi}_{I}\left(\sigma_{L}^{2}-\sigma_{R}^{2}+\epsilon_{I}\right)\left[\left(D_{L} \mathbf{u}\right)_{N}-\left(D_{R} \mathbf{v}\right)_{0}\right] \\
& +\left(D_{R} \boldsymbol{\varphi}_{R}\right)_{0}\left(\sigma_{L}^{3}-\epsilon_{I}-\sigma_{R}^{3}\right)\left[u_{N}-v_{0}\right]+\left(D_{R} \boldsymbol{\varphi}_{R}\right)_{0}\left(\sigma_{L}^{4}-\sigma_{R}^{4}\right)\left[\left(D_{L} \mathbf{u}\right)_{N}-\left(D_{R} \mathbf{v}\right)_{0}\right] \\
& +\left[\left(D_{L} \boldsymbol{\varphi}_{L}\right)_{N}-\left(D_{R} \boldsymbol{\varphi}_{R}\right)_{0}\right]\left[\sigma_{L}^{3}\left(u_{N}-v_{0}\right)-\epsilon_{I} u_{N}+\sigma_{L}^{4}\left(\left(D_{L} \mathbf{u}\right)_{N}-\left(D_{R} \mathbf{v}\right)_{0}\right)\right] .
\end{aligned}
$$

The last term in IT is proportional to the order of the scheme. Following [7], we say that (8) is conservative to the order of the scheme if $I T=0$, for all $\varphi_{L} \in V_{L}$ and $\varphi_{R} \in V_{R}$. This holds if $\left(D_{L} \varphi_{L}\right)_{N}=\left(D_{R} \varphi_{R}\right)_{0}$ and

$$
\sigma_{L}^{1}=\sigma_{R}^{1}, \quad \sigma_{L}^{2}=\sigma_{R}^{2}-\epsilon_{I}, \quad \sigma_{L}^{3}=\sigma_{R}^{3}+\epsilon_{I}, \quad \sigma_{L}^{4}=\sigma_{R}^{4}
$$

On the other hand, if (8) is conservative to the order of the scheme, then $I T=0$ for all possible solutions and test functions which implies that (13) must hold.

We summarize the result in 
Proposition 1. The interface procedure in (8) is conservative if and only if (13) holds.

Remark 2. It can be shown that Proposition 1 holds for narrow stencil SBP operators that can accommodate variable coefficients as defined in [8].

Next, we multiply (9) with $\boldsymbol{\Phi}_{L}^{T}\left(P_{L} \otimes I_{2}\right)$ and $\boldsymbol{\Phi}_{R}^{T}\left(P_{R} \otimes I_{2}\right)$ to get

$$
\begin{aligned}
& \boldsymbol{\Phi}_{L}^{T}\left(P_{L} \otimes \tilde{I}\right) \mathbf{U}_{t}+\boldsymbol{\Phi}_{R}^{T}\left(P_{R} \otimes \tilde{I}\right) \mathbf{V}_{t}+\boldsymbol{\Phi}_{L}^{T}\left(P_{L} \otimes I_{2}\right) \mathbf{A}_{L} \mathbf{U}+\boldsymbol{\Phi}_{R}^{T}\left(P_{R} \otimes I_{2}\right) \mathbf{A}_{R} \mathbf{V} \\
= & \boldsymbol{\Phi}_{I}^{T}\left(\Sigma_{l}-\Sigma_{r}-B\right)\left[U_{N}-V_{0}\right]+\boldsymbol{\Phi}_{L}^{T}\left(D_{L}^{T} P_{L} \otimes B\right) \mathbf{U}+\boldsymbol{\Phi}_{R}^{T}\left(D_{R}^{T} P_{R} \otimes B\right) \mathbf{V},
\end{aligned}
$$

which mimics the continuous formulation in (4). In (14), $\boldsymbol{\Phi}_{I}:=\left(\boldsymbol{\Phi}_{L}\right)_{N}=$ $\left(\boldsymbol{\Phi}_{R}\right)_{0}$, and a conservative scheme requires

$$
\Sigma_{l}=\Sigma_{r}+B .
$$

On the other hand, the interface term on the RHS of (14) must vanish for all possible solutions and test functions if (9) is a conservative scheme, which implies that (15) holds.

We summarize the result in

Proposition 2. The interface procedure in (9) is conservative if and only if (15) holds.

Remark 3. If the scheme (8) is conservative, then the first order scheme (9) with penalty matrices given in (10), is conservative. Similarly, if the scheme (9) is conservative, then the second order scheme (8) with penalty coefficients given in (11), is conservative.

Remark 4. Since $\sigma_{L}^{4}=\sigma_{R}^{4} \neq 0$ may increase the truncation error of the scheme (8), see [5], an obvious choice is $\sigma_{L}^{4}=\sigma_{R}^{4}=0$, which is used in the rest of the paper.

\subsection{Dual Consistency}

To determine dual consistency, we rewrite (8) in the compact form

$$
\mathbf{w}_{t}+L \mathbf{w}=0, \quad L=-\left(D_{2}+P^{-1} \Sigma\right),
$$

where $\mathbf{w}=[\mathbf{u}, \mathbf{v}]^{T}, D_{2}=\operatorname{diag}\left(D_{L} \boldsymbol{\epsilon}_{L} D_{L}, D_{R} \boldsymbol{\epsilon}_{R} D_{R}\right), P^{-1}=\operatorname{diag}\left(P_{L}^{-1}, P_{R}^{-1}\right)$ and

$$
\Sigma=F\left(\Sigma_{1}+D \Sigma_{2}\right)+D^{T} F\left(\Sigma_{3}+D \Sigma_{4}\right), \quad F=\left[\begin{array}{cc}
+E_{N} & -E_{I} \\
-E_{I}^{T} & +E_{0}
\end{array}\right] .
$$


In (17), $\Sigma_{i}=\operatorname{diag}\left(\sigma_{L}^{i}, \sigma_{R}^{i}\right)$, where $\sigma_{L, R}^{i}$ given in (8), $D=\operatorname{diag}\left(D_{L}, D_{R}\right)$, while $E_{N}=e_{N} e_{N}^{T}$ and $E_{0}=e_{0} e_{0}^{T}$. The $(N+1) \times(M+1)$ matrix $E_{I}=e_{N} e_{0}^{T}$ picks out the parts of the vectors residing at the interface such that $E_{I} \mathbf{v}=$ $\left[0, \cdots, 0, v_{0}\right]^{T}$ and $E_{I}^{T} \mathbf{u}=\left[u_{N}, 0, \cdots, 0\right]^{T}$.

The discrete dual problem corresponding to (16) is (see [6])

$$
\boldsymbol{\vartheta}_{\tau}+P^{-1} L^{T} P \vartheta=0,
$$

where $\boldsymbol{\vartheta}=[\boldsymbol{\phi}, \boldsymbol{\psi}]^{T}$. By using the SBP properties of the operators, we expand (18) and write it in component form as

$$
\begin{aligned}
& \boldsymbol{\phi}_{t}-D_{L}\left(\boldsymbol{\epsilon}_{L} D_{L} \boldsymbol{\phi}\right)=P_{L}^{-1}\left[\sigma_{L}^{1} \boldsymbol{\phi}_{N}-\sigma_{R}^{1} \boldsymbol{\psi}_{0}+\left(\sigma_{L}^{3}-\epsilon_{I}\right)\left(D_{L} \boldsymbol{\phi}\right)_{N}-\sigma_{R}^{3}\left(D_{R} \boldsymbol{\psi}\right)_{0}\right] e_{N} \\
& \quad+P_{L}^{-1} D_{L}^{T}\left[\left(\epsilon_{I}+\sigma_{L}^{2}\right) \boldsymbol{\phi}_{N}-\sigma_{R}^{2} \boldsymbol{\psi}_{0}\right] e_{N}, \\
& \boldsymbol{\psi}_{t}-D_{R}\left(\boldsymbol{\epsilon}_{R} D_{R} \boldsymbol{\psi}\right)=P_{R}^{-1}\left[\sigma_{R}^{1} \boldsymbol{\psi}_{0}-\sigma_{L}^{1} \boldsymbol{\phi}_{N}+\left(\epsilon_{I}+\sigma_{R}^{3}\right)\left(D_{R} \boldsymbol{\psi}\right)_{0}-\sigma_{L}^{3}\left(D_{L} \boldsymbol{\phi}\right)_{N}\right] e_{0} \\
& \quad+P_{R}^{-1} D_{R}^{T}\left[\left(\sigma_{R}^{2}-\epsilon_{I}\right) \boldsymbol{\psi}_{0}-\sigma_{L}^{2} \boldsymbol{\phi}_{N}\right] e_{0} .
\end{aligned}
$$

Dual consistency requires that (19) is a consistent approximation of (5), which leads to

Proposition 3. The scheme (19) is dual consistent if only if (13) holds.

Proof. The left-hand side (LHS) of (19) approximate the equations in (5). If (13) is satisfied, then the RHS of (19) imposes the dual interface conditions in (5). On the other hand, the interface terms on the RHS of (19) should vanish if $\varphi$ and $\psi$ satisfies the numerical interface conditions exactly. In other words, if (13) is not satisfied, the numerical scheme (19) would impose interface conditions at $x=0$ that do not exist in the adjoint problem.

To determine dual consistency, we rewrite (9) in compact form

$$
\tilde{\mathbf{I}} \mathbf{W}_{t}+\mathcal{L} \mathbf{W}=0, \quad \mathcal{L}=\mathcal{D}_{2}-\mathcal{P}^{-1} S, \quad \tilde{\mathbf{I}}=\operatorname{diag}\left(I_{N} \otimes \tilde{I}, I_{M} \otimes \tilde{I}\right),
$$

and $\mathbf{W}=[\mathbf{U}, \mathbf{V}]$. In $(20), \mathcal{D}_{2}=\operatorname{diag}\left(\mathbf{A}_{L}+D_{L} \otimes B, \mathbf{A}_{R}+D_{R} \otimes B\right)$, and

$$
S=\left[\begin{array}{ll}
+E_{N} \otimes \Sigma_{l} & -E_{I} \otimes \Sigma_{l} \\
-E_{I}^{T} \otimes \Sigma_{r} & +E_{0} \otimes \Sigma_{r}
\end{array}\right], \quad \mathcal{P}^{-1}=\operatorname{diag}\left(P_{L}^{-1} \otimes I_{2}, P_{R}^{-1} \otimes I_{2}\right) .
$$

The discrete dual problem corresponding to (20) is

$$
\tilde{\mathbf{I}} \boldsymbol{\theta}_{\tau}+\mathcal{P}^{-1} \mathcal{L}^{T} \mathcal{P} \boldsymbol{\theta}=0
$$


where $\boldsymbol{\theta}=[\boldsymbol{\Phi}, \boldsymbol{\Psi}]^{T}$. By expanding (21) in component form, we find

$$
\begin{aligned}
& \left(I_{N} \otimes \tilde{I}\right) \boldsymbol{\Phi}_{t}+\left[\mathbf{A}_{L}-D_{L} \otimes B\right] \boldsymbol{\Phi}=\left(P_{L}^{-1} e_{N} \otimes\left(\Sigma_{l}^{T}-B^{T}\right)\right) \boldsymbol{\Phi}_{N}-\left(P_{L}^{-1} e_{N} \otimes \Sigma_{r}^{T}\right) \boldsymbol{\Psi}_{0}, \\
& \left(I_{M} \otimes \tilde{I}\right) \boldsymbol{\Psi}_{t}+\left[\mathbf{A}_{R}-D_{R} \otimes B\right] \boldsymbol{\Psi}=\left(P_{R}^{-1} e_{0} \otimes\left(\Sigma_{r}^{T}+B^{T}\right)\right) \boldsymbol{\Psi}_{0}-\left(P_{R}^{-1} e_{0} \otimes \Sigma_{l}^{T}\right) \boldsymbol{\Phi}_{N} .
\end{aligned}
$$

Proposition 4. The scheme (22) is dual consistent if only if (15) holds.

Proof. The terms on the LHS in (22) approximate the two systems of equations in (6). If (15) is satisfied, the RHS of (22) imposes the dual interface conditions in (6). On the other hand, the interface terms on the RHS of (22) should vanish if $\boldsymbol{\Phi}$ and $\boldsymbol{\Psi}$ satisfies the numerical interface conditions exactly. In other words, if (15) is not satisfied, the numerical scheme (22) would impose interface conditions at $x=0$ that do not exist in the adjoint problem.

\section{Equivalence of conservation and dual consistency}

We are now ready to state the main results.

Proposition 5. The approximation (16) is conservative if and only if it is dual consistent.

Proof. It follows immediately from Propositions (1) and (3).

Proposition 6. The approximation (20) is conservative if and only if it is dual consistent.

Proof. It follows immediately from Propositions (2) and (4).

Remark 5. Proposition 5 and 6 imply that the conservation relations in Remark 3 also lead to dual consistency.

\section{References}

\section{References}

[1] J. Nordström, F. Ghasemi, On the relation between conservation and dual consistency for summation-by-parts schemes, Journal of Computational Physics 344 (2017) 437-439. 
[2] J. Nordström, F. Ghasemi, Corrigendum to "On the relation between conservation and dual consistency for summation-by-parts schemes", Journal of Computational Physics 360 (2018) 247.

[3] B. Cockburn, C.-W. Shu, The local discontinuous Galerkin method for time-dependent convection-diffusion systems, SIAM Journal on Numerical Analysis 35 (1998) 2440-2463.

[4] D. N. Arnold, F. Brezzi, B. Cockburn, L. D. Marini, Unified analysis of discontinuous Galerkin methods for elliptic problems, SIAM Journal on Numerical Analysis 39 (2002) 1749-1779.

[5] M. H. Carpenter, J. Nordström, D. Gottlieb, Revisiting and extending interface penalties for multi-domain summation-by-parts operators, Journal of Scientific Computing 45 (2010) 118-150.

[6] J. Berg, J. Nordström, Superconvergent functional output for timedependent problems using finite differences on summation-by-parts form, Journal of Computational Physics 231 (2012) 6846-6860.

[7] K. Mattsson, M. H. Carpenter, Stable and accurate interpolation operators for high-order multiblock finite difference methods, SIAM Journal on Scientific Computing 32 (2010) 2298-2320.

[8] K. Mattsson, Summation by parts operators for finite difference approximations of second-derivatives with variable coefficients, Journal of Scientific Computing 51 (2012) 650-682. 